\title{
Gibbs Thermodynamics of the Renninger-Wilemski Problem
}

\author{
Victor Kurasov \\ Faculty of Physics, Saint Petersburg University, Kamennoostrovskiy Prospekt 54/31, Office 60, Saint Petersburg 197022, Russia
}

Correspondence should be addressed to Victor Kurasov; victor_kurasov@yahoo.com

Received 5 August 2014; Accepted 2 January 2015

Academic Editor: Y. Peles

Copyright (C) 2015 Victor Kurasov. This is an open access article distributed under the Creative Commons Attribution License, which permits unrestricted use, distribution, and reproduction in any medium, provided the original work is properly cited.

\begin{abstract}
The Renninger-Wilemski problem in nucleation is analyzed. The Gibbs dividing surfaces method with external parameters is used to enrich the initial model. It is shown that both the traditional (Doyle) model and the Renninger-Wilemski model are not complete ones and, namely, the Gibbs dividing surface approach can solve this problem. It is shown that the application of the Gibbs approach also requires some model constructions. The simplified Gibbs model is proposed. It is shown that the simplified Gibbs model gives for the height of activation barrier the same numerical results as the Renninger-Wilemski model.
\end{abstract}

\section{Introduction}

Although the principles of thermodynamics are well grounded, there exist many narrow questions in applications. One of such questions belongs to the new phase embryos formation. Despite the fact that the free energy of the new phase embryo can be calculated in frames of equilibrium thermodynamics, there remain many questions of account of specific conditions for the critical embryo appearance.

There is no need to demonstrate the actuality of nucleation taking place practically overall. The importance of nucleation initiates investigations from Wilson's experiments [1-3] up to modern investigations. Thermodynamic aspects of investigations occupy an important place in this field considering not only the fundamental question of stationarity (see to clarify it, e.g., $[4,5]$ ), but also the problems of smallness of characteristic embryos and corrections appeared because of its sizes and shapes [6]. The results given by thermodynamics immediately lead to quantitative description of kinetics [7] and can be included into consideration of some rather complex aggregates with very specific properties [8]. This consideration is based mainly on thermodynamics of small embryos. Here we will discuss, namely, some important specific features of the small embryos thermodynamics.

The bright demonstration of specific features of thermodynamic aspects is the Renninger-Wilemski problem which occupies an important place in the nucleation of multicomponent liquids. An abbreviated version of this problem sounds as follows:

(i) Is it necessary to take the derivative of the surface tension on concentration to determine the characteristics of a critical droplet?

From the first glance this problem sounds so simple that the answer seems to be evident. But it took a dozens of years to clarify this problem.

This problem arises from the very beginning of investigations of the multicomponent nucleation since the pioneer paper written by Reiss [9], but until the paper of Renninger et al. [10] this problem was somehow in a shadow. The classical theory of nucleation [11, 12] is standing somehow aside of this question. The reason of this shadow is the rather weak dependence of the surface tension on concentration in the intermediate region of concentrations for the dominating majority of mixtures. The physical nature of this weakness is the enrichment of the surface layer with the surfaceactive molecules. When it is easy to find in the bulk of an embryo a molecule of the surface-active substance then it is profitable to catch it and to put in the surface. Roughly speaking only an entropy of mixing has to be paid here. The energy profit in this situation is a surface with a surface-active substance. So, the surface tension appears to be a relatively smooth function of concentration. Only when it is difficult to find such a molecule in a bulk liquid one has to pay some 
relatively essential work to find it and the surface tension begins to depend on concentration essentially. But the last situation occurs at small concentrations and this case should be preferably described as a one-component nucleation with some impurities.

The mentioned weak dependence allows many authors in 1950-80s to ignore the fact of dependence of the surface tension on concentration, although there exist some investigations like that by Mirabel and Katz [13]. In [13] the correction for the Young-Laplace formula with the derivative of the surface tension on concentration was presented. This correction is rather doubtful.

The statement that it is necessary to forbid to differentiate the surface tension was announced by Renninger and coauthors in [10] criticizing mainly Doyle [14] and Mirabel and Katz [13]. Before [10] the situation was the following: there exists the expression for the free energy, it is clear that the free energy of the critical embryo is the free energy at the saddle point, and it is clear that to get the coordinates of the saddle point it is necessary to take the free energy partial derivatives and to put them to zero, but no special attention was paid for the question what to do with the derivative of the surface tension on the solution concentration.

It was Doyle [15] who presented contrary to [10] the arguments for the traditional version with the differentiation of the free energy on concentration having justified his initial approach in [14]. Later Wilemski clarified the problem in [16, $17]$ and one could say after $[16,17]$ that the phenomenological recipe required that it was necessary to forbid to differentiate the surface tension. This seemed to be the final conclusion in this question. Since that time the problem is called the Renninger-Wilemski (RW) problem and seemed to be the closed problem in thermodynamics.

This problem was revitalized in 2003 by Reiss and Reguera $[18,19]$ who showed by a refinement of some connections for derivatives of chemical potentials in droplet the alternative variant of derivation of equations which are similar to those used by Dole. One can say that technically the publication $[18,19]$ was the top of analysis of this problem. But the Gibbs approach has not been considered in $[18,19]$. It was announced in $[18,19]$ that the Gibbs approach [20] "is of course the most sophisticated and general, but its application requires more information than is available in macroscopic thermodynamic observables." Meanwhile the recent investigations on thermodynamics of complex systems successfully operate with the quantities of excesses which is typical for the Gibbs dividing surfaces conception.

One has to mention that already after Wilemski in the field of multicomponent nucleation many models use the elements of the Gibbs dividing surface method but in their proper constructions. Instead of application of thermodynamics with some parameters of the model these papers try to build their "true" constructions without any choice of parameters. Here one can mention the approach of Debenedetti [21], the model of Nishioka and Kusaka [22], and the approach of Laaksonen et al. [23] which was analyzed also in [24]. All these models have the features of the Gibbs dividing surface method, but all of them propose some specific relations to close the system of equations. All of them announced the recipe for determination of the critical embryo characteristics as the only "true" one although the Gibbs model is principally a model with external parameters.

Here we will analyze both traditional models (the Doyle model and the Renninger-Wilemski model) and show that both of them are not self-consistent. The alternative model as realization of the Gibbs dividing surface method will be presented. In the last part it will be shown how to simplify this approach.

\section{Initial Remarks}

To determine the coordinates of the critical embryo one can follow at least two ways.

(i) The first way is to construct some expression (namely, the expression in the capillary approximation) for the total free energy of the critical embryo formation and then to determine the coordinates of the critical embryo as the coordinates of the free energy saddle point by differentiating and putting the derivatives to zero.

(ii) The second way is to use the general relations coming from the differential analysis such as the LaplaceYoung formula and the Gibbs-Thomson formula for the equilibrium (critical) droplet and to put into these formulas some concrete models for necessary values.

When the expression for the free energy is precise then both approaches have to give one and the same result. The trouble is that everything we can suggest is no more than an approximation. In fact these approaches historically led to two different results and this discrepancy has now the name of the Renninger-Wilemski problem (for such a terminology see $[18,19])$. Now it is clear that both results can be derived in both approaches but historically Renninger et al. [10] used the second approach and their opponent Doyle [15] used the first one. Following the history of the question we will speak about RW-model and about D-model correspondingly. Certainly, this classification is rather artificial.

The question of thermodynamic derivations of the surface characteristics was the subject of numerous investigations. The thermodynamic definition of the surface tension is discussed in [25]. Thermodynamics of a surface layer is analyzed in [26].

At first it is necessary to present the formal thermodynamic derivation in order to see where the hidden suppositions about the structure of the system under consideration have been done. Here we follow the manner of $[18,19]$ because, namely, that derivation opened the possibility to see different approaches in frames of the differential formalism. Certainly some essential modifications will be introduced.

Since in the system where the embryo is born the variables $T$ (temperature) and $V$ (volume) are supposed to be fixed, one has to use the Helmholtz free energy

$$
F=U-T S \text {. }
$$

Really, according to principles of thermodynamics,

$$
d F=-S d T-p d V .
$$


We suppose that the terms $\sum_{i} \mu_{i} N_{i}$ ( $\mu_{i}$ are the chemical potentials, $N_{i}$ are the numbers of particles, and index $i$ numerates the components) are included into thermodynamic potentials.

One has to split $U$ and $S$ into contributions from the liquid phase (index $l$ ) and the vapor phase (index $v)^{2}$. Then

$$
\begin{gathered}
U=U_{l}+U_{v}, \quad S=S_{l}+S_{v}, \quad V=V_{l}+V_{v}, \\
d U_{v}=T d S_{v}-p_{v} d V_{v}+\sum_{i} \mu_{v i} d N_{v i}, \\
d U_{l}=T d S_{l}-p_{l} d V_{l}+\sum_{i} \mu_{l i} d N_{l i} .
\end{gathered}
$$

Since

$$
d V_{l}=-d V_{v}, \quad d N_{v i}=-d N_{l i}
$$

we come to

$$
d U=T d S-\left(p_{l}-\widehat{p}_{v}\right) d V_{l}+\sum_{i}\left(\mu_{l i}-\mu_{v i}\right) d N_{l i} .
$$

Here the symbol $\widehat{\cdots}$ indicates that the pressure is the pressure under the curved surface; that is, the Young-Laplace correction $2 \gamma / r$ has to be added ( $\gamma$ is a surface tension, $r$ is a radius of a droplet). Then

$$
d F=-\left(p_{l}-\left(p_{v}+\frac{2 \gamma}{r}\right)\right) d V_{l}+\sum_{i}\left(\mu_{l i}-\mu_{v i}\right) d N_{l i}
$$

In the state of equilibrium we have $d F=0$ which gives

$$
p_{l}=p_{v}+\frac{2 \gamma}{r} .
$$

This is the Young-Laplace equation and

$$
\mu_{l i}\left[p_{v}+\frac{2 \gamma}{r}\right]=\mu_{v i}\left[p_{v}\right] .
$$

Here and later the square brackets show the functional dependence to avoid misreading as multiplication. If the index of variable is absent it means that the dependence takes place over the whole set of variables.

Now it is necessary to go in chemical potentials from $p$ to $p+2 \gamma / r$ explicitly. To do this it is necessary to calculate $\partial \mu_{l i} / \partial p$. To fulfil this transformation we take the Maxwell relations for the Gibbs free energy $G_{l}=U_{l}-T S_{l}+p_{l} V_{l}$ with a differential

$$
d G_{l}=-S_{l} d T+V_{l} d p_{l}+\sum_{i} \mu_{l i} d N_{l i} .
$$

Then

$$
\frac{\partial \mu_{i l}}{\partial p_{l}}=v_{l i}
$$

where $v_{l i}$ is the volume per one molecule of component $i$ in a liquid phase. We suppose the liquid to be incompressible. So, the rhs of the previous equation is constant and can be easily integrated. Equation (10) is of great importance since it will lead to the Gibbs-Thomson equation. Then

$$
\mu_{l i}\left[p+\frac{2 \gamma}{r}\right]=\mu_{l i}[p]+\frac{v_{l i} 2 \gamma}{r}
$$

and (8) looks like

$$
\Delta \mu_{i}=\frac{v_{l i} 2 \gamma}{r},
$$

where $\Delta \mu_{i}$ is the difference of chemical potentials at the external pressure in the system. The last relation is the so-called Gibbs-Thomson equation well known in thermodynamics.

Equation (12) leads to invariant

$$
\frac{\Delta \mu_{l i}}{v_{l i}}=\frac{\Delta \mu_{l j}}{v_{l j}}
$$

and allows a clear physical interpretation. Namely, (12) is the basis for RW-model.

Here it is necessary to make one very essential comment. Having written equation (9) for the differential of $G_{l}=U_{l}-$ $T S_{l}+p_{l} V_{l}$ we miss the term $\gamma A$, that is, the surface tension $\gamma$ multiplied on the surface area $A$. We can include this term and write

$$
d G_{l}=-S_{l} d T+V_{l} d p_{l}+\sum_{i} \mu_{l i} d N_{l i}+\gamma d A .
$$

This does not change the derivation. It is another moment which is important here. Beside this we have to note that ordinary it is implied that $\gamma \mathrm{A}$ is independent of other characteristics presented in (14). But in the droplet there is a connection between $A$ and $N_{l i}$. This puts a question on the validity of this derivation. It will be discussed below.

Equation (6) for $d F$ can be integrated from $N_{l i}=0$ up to the current size which gives $F\left[N_{i}\right]$. To fulfil this procedure it is convenient to present the dependence on $N_{l i}$ as the dependence on concentrations defined ${ }^{3}$ as

$$
\xi_{i}=\frac{N_{l i}}{N_{0}}
$$

where

$$
N_{0}=\sum_{i} N_{l i}
$$

is the total number of molecules in a drop.

As an extensive variable ${ }^{4}$ we can take $N_{0}$, but some other variants of choice are possible and will be presented below.

Instead of integration one can suggest to present the model for $F$ and then to check whether it leads to (7), (12) by differentiation. We are interested here, namely, in the last relations.

One can easily suggest the following expression for $F$ :

$$
F\left[N_{l i}\right]=F[0]-\sum_{i} \Delta \mu_{i} N_{l i}+\gamma A .
$$

Here and below $A$ is the surface area of the embryo. The term $V_{l}\left(p_{l}-p\right)$ is missed here since it is very small. It corresponds to 
the difference between the situation when the volume of the system is fixed or the pressure in the system is fixed. Concrete conditions ordinary are not well posed.

The condition for the equilibrium is

$$
\frac{\partial F}{\partial N_{l i}}=0
$$

The explicit differentiation has to be done with account of the Gibbs-Duhem equation for the bulk liquid

$$
\sum N_{l i} d \mu_{l i}+S_{l} d T-V_{l} d p=0
$$

which is actually

$$
\sum N_{l i} d \mu_{l i}=0
$$

The last relation will be satisfied automatically and inevitably when we use some concrete dependencies for chemical potentials in liquid.

The result of differentiation (we take for simplicity a twocomponent case) on account of

$$
A=C\left(\sum_{i} v_{l i} N_{l i}\right)^{2 / 3} \quad C=(36 \pi)^{1 / 3}
$$

is

$$
\frac{\partial F\left[N_{l i}\right]}{\partial N_{l i}}=-\Delta \mu_{i}+\frac{2 \gamma}{r}+\left(\frac{3 v_{m}\left(1-\xi_{i}\right)}{r}\right)\left(\frac{d \gamma}{d \xi_{i}}\right),
$$

where

$$
v_{m}=\sum \xi_{i} v_{l i}
$$

We see that the equilibrium condition

$$
\Delta \mu_{i}=\frac{2 \gamma}{r}+\left(\frac{3 v_{m}\left(1-\xi_{i}\right)}{r}\right)\left(\frac{d \gamma}{d \xi_{i}}\right)
$$

differs from the Gibbs-Thomson equation by the last term. This equation is the basis in D-model.

In RW-model one can also explain how to come to (12) starting from (17). In the differentiation of $F$ instead of (19) it is necessary to take the surface variant of the Gibbs-Duhem equation

$$
\sum N_{l i} d \mu_{l i}+S_{l} d T-V_{l} d p+A d \gamma=0
$$

which can be approximately reduced to

$$
\sum N_{l i} d \mu_{l i}+A d \gamma=0
$$

The last term in the previous equation cancels the derivative of the surface tension on concentration and we come to Gibbs-Thomson equations (12).

For a long time it seemed that (12) is preferable because there exists a differential method to see (12). But Reiss and Reguera showed that it is possible to get (24) in modification of the way we came here to (12). In integration $d \mu_{l i} / d p$ it is necessary to take into account not only the direct dependence $\partial \mu_{l i} / \partial p=v_{l i}$ but also the dependence on size of the embryo through the dependence

$$
\frac{\partial \mu_{l i}}{\partial A}=\frac{\partial \gamma}{\partial N_{l i}}
$$

The last relation can be found if we start with the Gibbs potential taking into account that in $U_{l}$ there already exits the surface term $\gamma$ A. Then

$$
d G_{l}=-S_{l} d T+V_{l} d p_{l}+\sum_{i} \mu_{l i} d N_{l i}+\gamma d A
$$

Then the Maxwell relation on variables $N_{l i}, A$ gives (27). Then the integration will lead precisely to (24). This derivation revitalizes D-model.

To complete the overview it is necessary to add the approach of Mirabel and Katz [13] who follow the differential way of consideration which led to (12). In order to get (24) Mirabel and Katz modified Young-Laplace equation (7) as

$$
p_{l}-p_{v}-\frac{2 \gamma}{r} \sim\left(\frac{d \gamma}{d \xi_{i}}\right)
$$

In [10] this approach was classified as an inappropriate one. We see that this approach is one of explanations how to reconcile the requirement to get the Gibbs-Kelvin equation and the requirement to have the free energy in the capillary model as described above. So, under the mentioned form of the free energy the true alternative is to modify the GibbsKelvin equation or to modify the Young-Laplace equation.

\section{Physical Model}

Ideas to find a tool outside the nucleation theory to solve the problem formulated above seem to be very attractive. But unfortunately there is no such tool. Speaking about the possibility of the direct measurement of the free energy of embryos one has to confess that except for some specific cases (see [27]) there is no direct mechanism to realize this possibility.

Certainly, practically every reliable model can be expressed in terms of statistic-mechanical approach (see [28]), but this can not be a tool to decide whether one can cancel the derivative of the surface tension. Here also both approaches can be justified on statistic-mechanical basis, the matter is which reference state of equilibrium is chosen.

One has to stress that the density functional theory cannot be the instrument to decide to cancel the derivatives $[29,30]$ because here the model form of the free energy is simply postulated at the level of the elementary hydrodynamic subsystems.

Unfortunately, the comparison with the 2D Ising model as it is proposed in [31] to solve the question of the necessity of cancelation cannot be fulfilled because one cannot generalize this model to the multicomponent case.

So, we have to formulate some physical model and discuss it. 
3.1. Different Meanings of the Surface Tension. One cannot argue that some excesses initiated by the interface have to be put into the theory. The most common one is the excess of the free energy, that is, the surface tension.

The answer to the question what approach is the most appropriate one lies in the method of construction of the capillary approximation and the expression for a free energy in this approximation. We have to reformulate a question: what is the surface tension which we will put in the surface term for the free energy of the embryo? Several answers are possible.

(i) This is the surface tension of an embryo.

Then the nucleation theory is the absolutely selfclosed theory and it cannot be checked. At the modern level of experimental devices there is no way to measure the surface tension of droplets otherwise than in the nucleation rate experiments. Everything which is measured in experiments is attributed to the value of surface tension according to theoretical dependencies derived in frames of an approach to calculate the nucleation rate.

(ii) This is the surface tension $\gamma_{t}$ observed in a capillary tube (index $t$ ) of the radius equal to the radius of the embryo (with corresponding wetting).

Here one can perform direct experiments to measure the surface tension. This is the way to construct a theory with the predicting force contrast to the previous case.

(iii) The surface tension is the plane surface tension $\gamma_{p}$. Certainly, this is a rather rough approximation, but ordinary, namely, the flat surface tension is known. By application of the methods of thermodynamics of small objects it is possible to construct some approximation for the surface tension in a tube and to reduce this case to the previous one. Also one has to mention that in some sense this case can be preferable because the surface excesses are mainly known in the case of the flat surface.

(iv) One can propose the conventional variant: $\gamma$ is the surface tension of a flat surface but at the surplus pressure (plus the Laplace-Young addition); this value can be found by interpolation from $P, T$ coexistence curve to surplus temperature or pressure.

There are no principal differences between this variant and the previous one. So, no special attention will be paid to this variant.

To go further one has to note that the capillary tube and the flat liquid are not a closed object as the droplet is. This is the inevitable difference and this difference will be important. The question is now the following:

(i) is it enough to account the compactness of the embryo only at the level of expression for $A$ as a function of the number of molecules inside the embryo or is it necessary to rewrite the analog of the Gibbs-Duhem equation which will be different from (25)?
Our conclusion is that there is no direct answer on this question in frames of macroscopic thermodynamics, but the principle of correspondence formulated below is rather reliable and solves this question.

3.2. Mean Chemical Potentials in Different Models. The object of further investigation will be the cluster of a liquid phase which is dense and compact contrast to a vapor phase. So, we will omit the index $l$, use $v_{i}$ instead of $N_{l i}$, and speak about chemical potentials instead of differences in chemical potentials ${ }^{5}$.

We will formulate the principle of correspondence.

(i) It is necessary to use the Gibbs-Duhem equation from the system which provides the surface tension used in the model. If we use the surface tension from a flat surface, we write the Gibbs-Duhem equation for a flat surface. If we use the surface tension from a capillary tube, we write the Gibbs-Duhem equation for a capillary tube.

Certainly, the most similar system with the explicitly measured surface tension for the situation of the embryo is the capillary tube. We take the surface tension from the capillary tube and have to write the Gibbs-Duhem equation for the capillary tube. This leads (as it will be shown later) to the Renninger-Wilemski recipe. But one has to stress that the principle of correspondence is no more than a supposition. Also it is necessary to add that one can act in a manner of the explicit attribution of the surface excesses which is typical for the Gibbs dividing surface approach.

In capillary models one has to use the surface tension from the situation with a capillary tube. It leads to the following consequence discussed below.

(i) In the droplet there is a strong connection between the radius $r$ and the number of molecules $\nu_{i}$; this connection is absent in the capillary tube.

The last connection can be written in a most simple version of the theory (without surface excesses taken into account) as

$$
\frac{4 \pi r^{3}}{3}=\sum_{i} v_{i} v_{l i} .
$$

If we use the Gibbs formalism there are surface excesses $\Psi_{i}$ of the substance (index $s$ marks the surface) and then

$$
\frac{4 \pi r^{3}}{3}=\sum_{i}\left(v_{i}-\Psi_{i}\right) v_{l i} .
$$

We attribute in frames of conception of a phase in the Gibbs method all other molecules to the bulk phase

$$
v_{b i}=v_{i}-\Psi_{i}
$$

(index $b$ marks the bulk region).

Approximately $\Psi_{i}$ are proportional to the surface area $4 \pi r^{2}:$

$$
\Psi_{i}=4 \pi r^{2} \rho_{i}
$$

where $\rho_{i}$ is the density of excess ${ }^{6}$. 
As the main dividing surface it is preferable to take the surface of tension where the Young-Laplace equation is precise.

Then

$$
\frac{4 \pi r^{3}}{3}=\sum_{i}\left(v_{i}-4 \pi r^{2} \rho_{i}\right) v_{l i}
$$

is an equation on $r$ as a function of $\nu_{i}$.

The concentrations are defined as

$$
\xi_{i}=\frac{v_{b i}}{\sum_{j} v_{b j}}
$$

It is a principal requirement that in the definition of concentration there stand the bulk numbers of molecules.

Now we will present constructions on the base of a capillary tube as a corresponding system for the free energy in capillary approximation. Until the place where the Gibbs model is formulated we forget about the excesses at the dividing surface. In the capillary tube there is no such balance relation as $v_{b i}=v_{i}-\Psi_{i}$. If one constructs the free energy of a capillary tube one gets

$$
F_{t}=\sum_{i} N_{i} \mu_{i}+\gamma_{t} A
$$

(index $t$ marks that we deal with a tube; $N_{i}$ are the numbers of molecules in a tube). Since the numbers $N_{i}$ are not fixed in principle (the tube is connected with a bath) one has to express the last relation through concentrations. This gives

$$
F_{t}=\sum N_{i} B_{b}[\xi]+\gamma A
$$

where $B_{b}$ is

$$
B_{b}=\sum \xi_{i} \mu_{i}[\xi]
$$

The value of $B_{b}$ has the sense of the averaged chemical potential. Consider

$$
B_{b}=\langle\mu\rangle
$$

The condition for the equilibrium will be

$$
\frac{d B_{b}}{d \xi}=0
$$

Since Gibbs-Duhem equation (19) takes place the last condition means

$$
\mu_{i}=\mu_{j}
$$

with a clear physical sense.

The same picture will be in a situation with a plane surface. The difference is that the surplus chemical potentials are counted here from the coexistence line, while in the case of a tube they are counted from the potentials in vapor phase under the Laplace pressure.

The term $\gamma$ A does not influence the concentration equilibrium when we suppose that the contact angle in a capillary tube is constant (namely, this situation is considered). Certainly, there exists a dependence on concentration through the increase of pressure, but we suppose that the dependence of activity coefficients, that is, the functions $f_{i}$ staying in standard expressions

$$
\mu_{i}=\ln \left(\frac{p}{p_{\mathrm{eq}}}\right)-\ln \xi_{i}-\ln f_{i}\left[\xi_{i}\right]
$$

on pressure, is small. Here $p_{\mathrm{eq}}$ is the pressure over a pure plane liquid phase and $\ln \left(p / p_{\text {eq }}\right)$ is a logarithm of the supersaturation plus one.

The situation in a droplet is another one. The surface area $A$ depends on $N_{i}$ and we have to take this dependence into account. There are several ways how to do it.

To see the preferable way we will analyze the structure of the free energy in the embryo. We present $F$ from (17) in the form like that in the one-dimensional case:

$$
F=-B_{d} \Omega+\Omega^{2 / 3}
$$

Here $\Omega$ is defined in order that $\Omega^{2 / 3}$ is the surface surplus input into the droplet free energy

$$
\Omega^{2 / 3}=A \gamma \text {. }
$$

For $B_{d}$ one can get on the base of (17):

$$
B_{d}=C^{-3 / 2} \gamma^{-3 / 2}\left(\frac{B_{b}}{v_{m}}\right),
$$

where $v_{m}$ is the mean volume of a molecule in a droplet.

The value of $B_{d}$ allows the following interpretation:

$$
B_{d} \sim \frac{\langle\mu\rangle}{\gamma^{3 / 2}\langle v\rangle}
$$

and one can consider the denominator in the last expression as the mean surface tension for one molecule in a power $3 / 2$ (certainly, one molecule has no surface tension).

Condition for an equilibrium concentration $\xi_{c}$ will be

$$
\frac{d B_{d}}{d \xi_{i}}=0
$$

Condition for an equilibrium when the derivative of $\gamma$ is put to zero will be

$$
\frac{d\left(B_{b} / v_{m}\right)}{d \xi_{i}}=0 .
$$

Here one can also get the interpretation for $B_{b} / v_{m}$ as the ratio of two mean values $\langle\mu\rangle=\mu_{m}$ and $\langle v\rangle=v_{m}$.

Both conditions are independent of the value of $\Omega$ and it is important. When we choose as a variable which is supplementary to $\xi$ any other variable we can come to the dependence of $\xi_{c}$ on $\Omega$ but at the saddle point the result will be the same.

We will call $B_{d}$ the mean chemical potential in the droplet in D-model and $B_{\mathrm{RW}} \sim B_{b} / v_{m}$ the mean chemical potential in the droplet in RW-model.

The property of independence on $\gamma$ of the minimum of $B_{b} / v_{m}$ is the serious argument for RW-model and against Dmodel. 
(A1) In the capillary tube which gives the tension used in the capillary approximation for $F$ there exists the same independence; the concentration is the argument which provides the minimum of $B_{b}$. The position of this minimum is independent of $\gamma$.

Immediately there arise two arguments for D-model against RW-model.

(A2) The number of molecules and the surface term $\gamma A$ ( $\gamma$ is the surface tension, $A$ is the surface area) are not independent in droplet. dent.

In the tube the situation is opposite. They are indepen-

When the molecule comes to the droplet it enlarges the volume and, thus, the surface term $\gamma$ A. In the tube such effect is absent. a tube ${ }^{7}$

So, here appears a question why we will use the model of

Even when the molecule does not change the volume of an embryo (e.g., this molecule is changed by the molecule of another type but with the same volume) the surface tension will be changed. We come to the following conclusion.

(A3) The composition of the surface layer has to be chosen not only to minimize the surface tension as it is in the tube but to minimize the whole surface term $\gamma A$; that is, the composition of the bulk region also acts on $A$ and, thus, on the surface term in a way different from the case of a tube.

This argument strengthens the conviction not to use the tube as the source of approximation for $F$ but to use directly D-model.

But the most important argument comes from the analysis of the Gibbs-Duhem relation. It is formulated below. The problem staying there is that the Gibbs-Duhem relation has to be used inevitably in any model, but to write it one has to adopt also some model. This model has the direct influence on the result.

3.3. Interpretation of the Gibbs-Duhem Relation. The principle of correspondence states that since we use the surface tension from the measurements with a capillary tube we have to use the Gibbs-Duhem relation for the surface of a tube. The Gibbs-Duhem relation for the surface in a tube has the following form ${ }^{8}$ :

$$
d \gamma+\sum \rho_{i} d \mu_{i}\left[\xi_{b i}\right]=0 .
$$

One can easily note that if

$$
\frac{\rho_{i}}{\sum_{j} \rho_{j}}=\xi_{b i}
$$

then

$$
\sum_{i} \rho_{i} d \mu_{i}\left[\xi_{b i}\right] \equiv 0
$$

and the derivative of the surface tension cannot be compensated $^{9}$.

So, the physical reason of the compensation of the derivative of the surface tension on the concentration is the difference of the concentration in the surface layer from the bulk concentration. Surface enrichment as a complication for consideration was noticed in [32], but there the different possibilities to formulate the reference system were not even mentioned.

Really, in any surface layer (let it be the layer of an imaginary thickness $l$ ) we see that the volume of the layer is approximately $l A$ and there are

$$
\frac{\xi_{b i} l A}{v_{m}}+\rho_{i} A
$$

molecules of a sort $i$. So the concentration $\xi_{s i}$ in a surface layer will be

$$
\xi_{s i}=\frac{\xi_{b i} l / v_{m}+\rho_{i}}{\sum_{j} \xi_{b j} l / v_{m}+\rho_{j}}
$$

and it does not coincide with $\xi_{b i}$. The difference between $\xi_{b i}$ and $\xi_{s i}$ explains why segregation is size dependent in diluted solutions, as in droplets of small size there is no sufficient number of atoms. Certainly, $l$ is a parameter and $\xi_{s i}$ cannot be calculated explicitly. But under any $l$ the value $\xi_{s i}$ does not coincide with $\xi_{b i}$.

We see that $\mathrm{D}$-model is the model with uniform liquid and RW-model is the model with nonuniform liquid. The account of nonuniformity of liquid in frames of RW-model is the simplest one but we see that this account is very important and makes the model more complete. It is rather difficult to realize that RW-model is the model with nonuniform liquid because the region of enrichment is not described explicitly. Also there are no explicit values of excesses which also can show the nonuniformity. The Gibbs-Duhem equation is the only trace of such nonuniformity. It is simply a necessary condition of enrichment in the model.

We come to the following argument.

(A4) Having used $\gamma$ from the situation with capillary tube of flat surface where the surface tension region is enriched with the surface-active component it is reasonable to take the model where the surface region is enriched according to the same rule, that is, according to Gibbs-Duhem equation (49).

This argument is the decisive argument in a choice favorable for RW-model.

Despite the choice of RW-model as a more adequate model we see that both models are not free from objections. For the RW-model the arguments A2 and A3 are the features ignored by this model. For the D-model the arguments $\mathrm{Al}$ and A4 are the unresolved problems. Below the Gibbs dividing surface method (G-model) will be presented and this method is more sophisticated than RW- and D-models. But still the Gibbs dividing surface method (at least at the level of the several first terms of decomposition) is no more than a model because the arguments $\mathrm{A} 2$ and $\mathrm{A} 3$ are not fully resolved. But 
it is the best model one can propose at the relatively simple level.

The terminology "Gibbs model" or "Gibbs dividing surface method" means nothing without any concrete recipe of approximations. Certainly, if we can get the characteristics of the curved surface of the closed confined droplet then the description becomes precise. The problem is that we do not know these characteristics; the optimal thing we can do is to use the characteristics of the curved surface over the unbounded system like the capillary tube is. The transmission of these characteristics to the situation of a droplet means the usage of the model. Evidently the capillary model is rather accurate and natural. But it is still a model. Here lies the source of discrepancy between theory and experiments on nucleation which tortures this field more than half a century. Certainly, this difficulty takes place in one-component case also.

\section{Gibbs Method}

The more adequate model than $\mathrm{D}$ - and RW-models can be constructed on the base of surface excesses and it will be done in this section.

First of all it is necessary to stress that we speak about the simplest vulgar version of the Gibbs dividing surfaces method. Namely, such a version is necessary to be installed instead of D-model or RW-model because the information about excesses even in a flat case is rather poor.

The structure of the Gibbs dividing surface method in the simplest (zero) approximation requires to consider the surface (it is more convenient to consider the surface of tension, i.e., the surface where the Young-Laplace formula takes place precisely) and the surface excesses of all components $\widetilde{N}_{s i}$ which will be marked by $\Psi_{i}$. Then the surface area $A$ will be calculated on the base of

$$
A=C\left(\sum_{i} v_{l i}\left(v_{i}-\Psi_{i}\right)\right)^{2 / 3}
$$

which forms an equation on $A$ :

$$
A=C\left(\sum_{i} v_{l i}\left(v_{i}-A \rho_{i}\right)\right)^{2 / 3}
$$

The first iteration is sufficient:

$$
A=C\left(\sum_{i} v_{l i}\left(v_{i}-C\left(\sum_{i} v_{l i} v_{i}\right)^{2 / 3} \rho_{i}\right)\right)^{2 / 3}
$$

We again recall that here and later we suppose the liquid to be incompressible and the volumes $v_{l i}$ to be independent of concentration.
From the first glance it seems that the free energy $F$ has to be approximately transformed from $F=\sum_{i} \mu_{i} \nu_{i}+\gamma A$ to

$$
\begin{aligned}
F= & \sum_{i} \mu_{i} \nu_{i} \\
& +\gamma\left(C\left(\sum_{i} v_{l i}\left(v_{i}-C\left(\sum_{i} v_{l i} v_{i}\right)^{2 / 3} \rho_{i}\right)\right)^{2 / 3}\right) .
\end{aligned}
$$

But actually this transformation can be avoided by use of special variables as it will be clear below.

One can fulfil the same analysis as above but instead of $\Omega$ one has to choose

$$
\Omega \longrightarrow \gamma^{3 / 2}\left(C\left(\sum_{i} v_{l i}\left(v_{i}-C\left(\sum_{i} v_{l i} v_{i}\right)^{2 / 3} \rho_{i}\right)\right)^{2 / 3}\right)^{3 / 2} .
$$

Then

$$
F=-\sum_{j} \lambda_{j} \mu_{j}-\sum_{j} \varrho_{j} \mu_{j} \frac{\Omega^{2 / 3}}{\gamma(\xi)}+\Omega^{2 / 3}
$$

with

$$
\lambda_{i}=v_{i}-\Psi_{i}
$$

Certainly,

$$
\frac{\lambda_{i}}{\lambda_{j}}=\frac{\xi_{i}}{\xi_{j}}
$$

One can choose instead of $\Omega$ the variable $\kappa$ by the following formula:

$$
\kappa=A^{3 / 2}\left(\gamma-\sum_{i} \varrho_{i} \mu_{i}\right)^{3 / 2} .
$$

In these variables the free energy $F$ has the form

$$
F=-\kappa b_{g}(\xi)+\kappa^{2 / 3}
$$

with the generalized chemical potential

$$
b_{g}=\frac{\sum_{i} \lambda_{i} \mu_{i}}{\kappa}
$$

or

$$
b_{g}=\sum_{i} \xi_{i} \mu_{i} \frac{\sum_{j} \lambda_{j}}{\kappa} .
$$

One has to show that $b_{g}$ does not depend on $\kappa$. To fulfill this derivation one can come to

$$
b_{g}=\sum_{i} \xi_{i} \mu_{i} \frac{\sum_{j} \lambda_{j}}{A^{3 / 2}\left(\gamma-\sum_{k} \varrho_{k} \mu_{k}\right)^{3 / 2}}
$$


or $^{10}$

$$
b_{g}=\sum_{i} \xi_{i} \mu_{i} \frac{\sum_{j} \lambda_{j}}{\left(\gamma-\sum_{k} \varrho_{k} \mu_{k}\right)^{3 / 2} \sum_{l} v_{l} \lambda_{l}} .
$$

It can be also presented as

$$
b_{g}=\sum_{i} \xi_{i} \mu_{i} \frac{1}{\left(\gamma-\sum_{k} \varrho_{k} \mu_{k}\right)^{3 / 2} \sum_{l} v_{l} \xi_{l}} .
$$

The last relation evidently shows that $b_{g}$ is really a function of $\xi$. The dependence on $\kappa$ is absent.

Formula (63) is valid for the arbitrary Gibbs model. This form will be called the canonic representation. Now we see that it takes place for the arbitrary model in frames of the Gibbs dividing surface method. This result is of great importance. Directly from this result follows the Gibbs rule. It means that the free energy of the critical embryo is one-third of the surface energy. Now this rule is shown for the arbitrary model from the Gibbs dividing surface method.

One can use expression (68) to clarify the RenningerWilemski problem. According to the Gibbs absorption relation $^{11}$

$$
d \gamma=\sum_{j} \varrho_{i} d \mu_{i}
$$

the derivative of the surface tension on concentration is canceled by the corresponding derivatives of $\varrho_{i}$ on $\xi$. So, if we write $b_{g}$ without surface excesses as

$$
b_{g}=\sum_{i} \xi_{i} \frac{1}{\gamma^{3 / 2} \sum_{j} v_{j} \xi_{j}}
$$

we have to forbid the differentiation of $\gamma$ on concentration. We see that in frames of the Gibbs approach (G-model) the Renninger-Wilemski problem is explained. Here there are no artificial requirements not to differentiate the surface tension on concentration. It is necessary to stress that the reason is not the formal Gibbs absorption equation, but the difference of concentrations in the bulk solution from the integral values.

One has also to recall that in frames of G-model there is one undefined parameter because in the $L$ component case there are only $L-1$ independent equations:

$$
\frac{d \gamma}{d \xi_{b i}}=\frac{\sum_{j} \rho_{j} d \mu_{j}}{d \xi_{b i}} .
$$

One additional parameter in G-model is inevitable. Ordinary it is the sum of excesses with some weights.

\section{The Reduced Gibbs Model}

The G-model is wider than the D-model and the RWmodel. It is important that G-model is the self-consistent one. But there exists one additional parameter which cannot be calculated. Namely, for two excesses $\rho_{1}$ and $\rho_{2}$ there exists only one equation:

$$
\frac{\rho_{1} d \mu_{1}}{d \xi_{b}}+\frac{\rho_{2} d \mu_{2}}{d \xi_{b}}=\frac{d \gamma}{d \xi_{b}}
$$

For $L$-component case there exist $L-1$ equations:

$$
\frac{\sum_{j} \rho_{j} d \mu_{j}\left[\xi_{b}\right]}{d \xi_{b i}}=\frac{d \gamma}{d \xi_{b i}}
$$

and the last $L$ th equation

$$
\frac{\sum_{j} \rho_{j} d \mu_{j}\left[\xi_{b}\right]}{d \xi_{b L}}=\frac{d \gamma}{d \xi_{b L}}
$$

is the superposition of the previous $L-1$ equations. So, it is necessary to add one equation. When one knows the distance $l$ between the surface of tension and the total equimolecular surface determined as

$$
\sum_{j} v_{j} v_{l j}=\left(\frac{4 \pi}{3}\right) r^{3}
$$

(certainly, it is possible to account the density in a vapor phase) then $(l \ll r)$

$$
\sum A \rho_{i} v_{l i}=A l .
$$

The most simple variant is to say that the total equimolecular surface coincides with the surface of tension. This corresponds to initial relation (30). It gives

$$
\sum \rho_{i} v_{l i}=0 .
$$

Certainly, this is artificial requirement and generally speaking it is wrong. The only justification is that this requirement is the simplest one and that it corresponds to the recipe (30) of the simplest and the oldest capillary approximation which is used since [9]. In [23, 24] this connection was announced to be the only true one. One has to stress that according to the Gibbs dividing surface method this connection has no preferences.

The main conclusion for the SG-model (simplified Gibbs model) is that the results for the free energy of the critical embryo, for concentration $\xi_{b}$ and for the variable $\Omega$ (here $\kappa$ ), coincide with results of RW-model.

The last statement can be easily checked by comparison of corresponding formulas.

The coincidence of the free energy of the critical embryo, the concentration and the surface of the critical embryo in both (RW and SG) models, does not mean that all characteristics coincide. In RW-model one can get

$$
v_{i}=\frac{\xi_{b i} \Omega}{v_{m} \gamma^{3 / 2} C^{3 / 2}}=N_{b i} .
$$

Certainly, to see the critical numbers $v_{c i}$ of the molecules of $i$ th component in SG-model one has to solve the system of equations and add $\rho_{i} 4 \pi r^{2}$ to

$$
v_{b i}=\frac{\xi_{b i} \Omega}{v_{m} \gamma^{3 / 2} C^{3 / 2}} .
$$

Then

$$
\nu_{i}=\frac{\xi_{b i} \Omega}{v_{m} \gamma^{3 / 2} C^{3 / 2}}+\rho_{i} 4 \pi r^{2}
$$


or

$$
v_{i}=\frac{\xi_{b i} \Omega}{\left(v_{m} \gamma^{3 / 2} C^{3 / 2}\right)}+\rho_{i} \Omega^{2 / 3} \gamma^{-1}
$$

and the result slightly differs from RW-model.

But this difference means absolutely nothing for the height of activation barrier. For the rate of nucleation since the change of the Zeldovich factor is negligible the error is negligible also.

We come to the same functional dependencies but with slightly changed values of coefficients. Such simple dependencies are probably the source for the simple scaling factors like it is observed in [33]. One can also mention that the applicability of the scaling approach to the free energy like the Fisher droplet model and analogous ideas by Stauffer [34] and later by Bauchspiess and Stauffer [35] can be also interpreted as the result of the simple scaling observed here. They can be also included in this picture by some moderate transformations.

\section{Self-Consistency of Thermodynamic Approaches}

From the first glance the situation with the RenningerWilemski problem is strange; there are no evident errors in both D-and RW-models, but at least one result is wrong. Does it mean that in construction of thermodynamic theories it is possible to make an "invisible error"? Fortunately it will be shown below that the difference in results for the free energy of the critical embryo obtained in different models has the order of correction.

To see this smallness one can redefine $\kappa$ as $A^{3 / 2}$ and forget about excesses. The value of $\kappa$ is the big parameter of the theory. Really, the thermodynamic description is valid only at great number of molecules in the embryo which requires a big value of $\kappa$.

Then $F=-B_{A} \kappa-\gamma \kappa^{2 / 3}$ and it is important that $B_{A} \sim$ $B_{b} / v_{m}$ does not contain $\gamma$. Really, from equation

$$
\left.\frac{\partial F}{\partial \xi}\right|_{\kappa=\text { fixed }}=\frac{\partial \gamma}{\partial \xi} \kappa^{2 / 3}-\frac{\partial B_{A}}{\partial \xi} \kappa
$$

it is seen that the first term $(\partial \gamma / \partial \xi) \kappa^{2 / 3}$ with the derivative $\partial \gamma / \partial \xi$ has the correction order $\kappa^{2 / 3}$ :

$$
\frac{\partial \gamma}{\partial \xi} \kappa^{2 / 3} \sim \kappa^{2 / 3}
$$

in comparison with the second term $\left(\partial B_{A} / \partial \xi\right) \kappa$ having the order $\kappa$ :

$$
\frac{\partial B_{A}}{\partial \xi} \kappa \sim \kappa
$$

It means that the correction will be small.

We extract this result because of its importance for the reconstruction of the logical self-consistency of thermodynamics. Only the correcting order of the term with the derivative of the surface tension allows to ignore it in the main order and to return the leading role of the ordinary capillary approximation.

Certainly, in situations of practical realization in experiments the difference between the order $\kappa$ and $\kappa^{2 / 3}$ can be hardly seen.

Since the formal recipe to resolve the RenningerWilemski problem is to forbid the differentiation of $\gamma$ on concentration then the equation on concentration will be different. It would cause the impression that there is a shift in a leading term. The true answer is that this result causes the shift in $F_{c}$ which has a correction order as it follows from the last equation.

One can treat the surface tension as a coefficient in the first correction term proportional to the surface of the embryo. The coefficients at $\kappa^{1 / 3}, \ln \kappa, \kappa^{-1 / 3}$, and so forth depend on intensive variables (concentration is one example). Their derivatives will be canceled by derivatives of corresponding excesses. The structure will resemble the RenningerWilemski problem. But here the dimension of "surface" will be $\kappa^{1 / 3}, \ln \kappa, \kappa^{-1 / 3}$, and so forth. This effect will be called the "generalized cancelation of derivatives on intensive variables." The considerations to justify the cancelation are practically the same as those presented here for the leading correction.

The necessity to develop the theory with surface excesses is evident because the surface excesses will essentially shift the position of the near-critical region in comparison with the size of the near-critical region. In RW-model the form of the near-critical region can not be explicitly described. But this region is extremely important for solution of kinetic equation to get the rate of nucleation.

The Gibbs method allows to get the form of the nearcritical region. Rigorously speaking to determine the form of the near-critical region one has to take the expression for $F$ with correction terms up to the order which causes the shift of position of the near-critical region. Now it is clear that the effect results mainly in the shift of the near-critical region while the form of the near-critical region remains approximately the same.

\section{Results and Conclusions}

The main new results of the current analysis are the following:

(i) It is shown that both D-model and RW-model are not complete ones.

(ii) The principle of correspondence is formulated. It is shown that this postulate leads to the RW-model.

(iii) It is shown that canonic representation takes place in the arbitrary Gibbs model.

(iv) The Gibbs rule is shown for the arbitrary model in the class of the Gibbs dividing surface method.

(v) The driving physical reason of RW problem is shown. It is the difference of concentration in a surface layer from the concentration in a bulk liquid phase.

(vi) The self-consistency of thermodynamic models is shown. 
Does the performance of this analysis mean that the free energy of the critical embryo in nucleation has been found? To comment on this question one has to note that the conditions of applicability of thermodynamic approach to solve the RW problem are very strong. Namely, ordinary it is required that

$$
v_{i} \gg 1 .
$$

But also the surface layer has to be the uniform media which requires

$$
\Psi_{i}=A \rho_{i} \gg 1 .
$$

The last inequality is very hard to satisfy. The difficulty occurs not only because the surface layer has the small volume (we mean the real surface layer which has the finite thickness and it is only reflected in $\Psi_{i}$ in Gibbs model) but also because there exists an effect of enrichment of the surface layer by the so-called surface active substances. Then there are only few molecules of the surface passive substance. This breaks the last inequality for the surface-passive components for rather big critical embryos. Otherwise we have to construct the theory with a nonuniform surface layer, which is extremely difficult to do, even approximately.

The theoretical constructions presented above can be generalized. In fact there exist several model methods to improve the construction of the free energy, for example, the Debenedetti Reiss method, discussed in [36]. These refinements can be inserted into the theory presented here without radical rearrangements.

One can refine the theory by inserting the dependence of the surface tension on radius (see, e.g., $[37,38]$ ). The special effects which appeared in the constrained systems (see [39]) are not taken into account here but they can be easily introduced into this theory. One can add that the possibility of deformation of the center of the embryo can be also taken into account. This effect is quite analogous to the deformation of heterogeneous centers and it was considered in [40].

We keep aside the possibility of fluctuations widely investigated theoretically and observed experimentally [41]. They can be also included into consideration without essential transformation of the theory. One can insert here the Tolmanlike corrections as well as the scaled expansions introduced in [42]. One has to note that any refinements like $[43,44]$ of the classical nucleation theory can be included here directly without any interference with results of this consideration. Refinements have to be put instead of the classical expression.

However, in this analysis we do not analyze the possibility that the bulk structure of the embryo can differ from the bulk structure of the reference bulk system in a capillary tube (for the principal possibility of such an effect see [45]). We consider the bulk of the embryos as the homogeneous media; for nonuniform density one can see [46]. The theory can be transformed to grasp this case also.

We do not pretend to go close to the critical point although the difference between concentrations in bulk and in surface layers can be interpreted in analogous manner. It seems that any extensions of the classical approach like [47] should be replaced by theoretical constructions based on the renormalized group approach.

\section{Conflict of Interests}

The author declares that there is no conflict of interests regarding the publication of this paper.

\section{Endnotes}

1. One can also suppose that the fixed variables are the temperature $T$ and the pressure $p$ and use the Gibbs free energy

$$
G=F+p V .
$$

The difference in the work of the droplet formation will be in a value of the volume of the appeared droplet multiplied on differences in pressure. Ordinary this term is very small and it is neglected. In the closed systems during the process of nucleation from diluted solution the concentration decreases. This effect is rather small for critical embryos and begins to be essential at big supercritical formations of a new phase. The corresponding effect in the global kinetics of nucleation is described in [48].

2. Already here we have to adopt some model: to divide $U$ and $S$ into two parts. Also we suppose that the temperature is one and the same for both parts; that is, we consider the isothermal nucleation. At this level of narration we do not need the surface excesses; these excesses will appear later.

3. Here this statement used in $[18,19]$ is not absolutely precise and will be corrected.

4. This variable used in $[18,19]$ as an extensive variable is only asymptotically extensive one which will be discussed.

5. Here we are not interested any more in the density of environment and denote the number of molecules inside the droplet by $v_{i}$. This notation marks that we are not interested in the number of molecules in a vapor phase.

6. Later it may be useful to write another coefficient of proportionality as $\Psi_{i}=A \rho_{i}$.

7. Namely, the Gibbs dividing surface for a tube leads to the cancelation of $d \gamma / d \xi_{i}$.

8. For the embryo we cannot extract the surface explicitly as an independent object since there is the connection (30). So, the base for such type of the Gibbs-Duhem relation is absent. Instead of this one has to express $A$ via the numbers of molecules taking into account relation (27).

9. Since in reality it is compensated it means that this possibility cannot be realized.

10. We miss $C$ for simplicity.

11. The sign is changed because of specific choice of differences of chemical potentials. 


\section{References}

[1] C. T. R. Wilson, "Condensation of water vapour in the presence of dust-free air and other gases," Philosophical Transactions A, vol. 189, no. 11, pp. 265-307, 1898.

[2] C. T. R. Wilson, "On the condensation nuclei produced in gases by the action of roentgen rays, uranium rays, ultraviolet rays and other agents," Philosophical Transactions of the Royal Society of London, vol. 192, no. 9, pp. 403-453, 1899.

[3] C. T. R. Wilson, "On the comparison effeciency as condensation nuclei of positively and negatively charged ions," Philosophical Transactions of the Royal Society A, vol. 193, p. 289, 1900.

[4] V. V. Ryazanov, "Nonequilibrium thermodynamics based on the distributions containing lifetime as a thermodynamic parameter," Journal of Thermodynamics, vol. 2011, Article ID 203203, 10 pages, 2011.

[5] V. V. Ryazanov, "Nonequilibrium thermodynamics and distributions time to achieve a given level of a stochastic process for energy of system," Journal of Thermodynamics, vol. 2012, Article ID 318032, 5 pages, 2012.

[6] R. Kumar, G. Sharma, and M. Kumar, "Effect of size and shape on the vibrational and thermodynamic properties of nanomaterials," Journal of Thermodynamics, vol. 2013, Article ID 328051, 5 pages, 2013.

[7] S. Turmanova, S. Genieva, and L. Vlaev, "Kinetics of nonisothermal degradation of some polymer composites: change of entropy at the formation of the activated complex from the reagents," Journal of Thermodynamics, vol. 2011, Article ID 605712, 10 pages, 2011.

[8] E. Hernández-Lemus, "Nonequilibrium thermodynamics of cell signaling," Journal of Thermodynamics, vol. 2012, Article ID 432143, 10 pages, 2012.

[9] H. Reiss, "The kinetics of phase transitions in binary systems," The Journal of Chemical Physics, vol. 18, no. 6, pp. 840-848, 1950.

[10] R. G. Renninger, F. C. Hiller, and R. C. Bone, "Comment on 'self-nucleation in the sulfuric acid-water system,' The Journal of Chemical Physics, vol. 75, no. 3, pp. 1584-1585, 1981.

[11] A. C. Zettlemoyer, Nucleation, Dekker, New York, NY, USA, 1969.

[12] F. F. Abraham, Homogeneous Nucleation Theory, Academic Press, New York, NY, USA, 1974.

[13] P. Mirabel and J. L. Katz, "Binary homogeneous nucleation as a mechanism for the formation of aerosols," The Journal of Chemical Physics, vol. 60, no. 3, pp. 1138-1144, 1974.

[14] G. J. Doyle, "Self-nucleation in the sulfuric acid-water system," The Journal of Chemical Physics, vol. 35, no. 3, p. 795, 1961.

[15] G. J. Doyle, "Response to the comment on 'Self-nucleation in the sulfuric acid-water system," The Journal of Chemical Physics, vol. 75, no. 3, p. $1585,1981$.

[16] G. Wilemski, "Composition of the critical nucleus in multicomponent vapor nucleation," The Journal of Chemical Physics, vol. 80, no. 3, p. 1370, 1984.

[17] G. Wilemski, "Revised classical binary nucleation theory for aqueous alcohol and acetone vapors," The Journal of Physical Chemistry, vol. 91, no. 10, pp. 2492-2498, 1987.

[18] H. Reiss and D. Reguera, "Nucleation in confined ideal binary mixtures: the Renninger-Wilemski problem revisited," Journal of Chemical Physics, vol. 119, no. 3, pp. 1533-1546, 2003.

[19] H. Reiss, Methods of Thermodynamics, Dover, Mineola, NY, USA, 1996.
[20] J. W. Gibbs, The Collected Works of J.W. Gibbs Longmans, Green, New York, NY, USA, 1928.

[21] P. G. Debenedetti, Metastable Liquids: Concepts and Principles, Princeton University Press, Princeton, NJ, USA, 1996.

[22] K. Nishioka and I. Kusaka, "Thermodynamic formulas of liquid phase nucleation from vapor in multicomponent systems," The Journal of Chemical Physics, vol. 96, no. 7, pp. 5370-5376, 1992.

[23] A. Laaksonen, R. McGraw, and H. Vehkamäki, "Liquid-drop formalism and free-energy surfaces in binary homogeneous nucleation theory," Journal of Chemical Physics, vol. 111, no. 5, pp. 2019-2027, 1999.

[24] Y. S. Djikaev, I. Napari, and A. Laaksonen, "On the closure conjectures for the Gibbsian approximation model of a binary droplet," The Journal of Chemical Physics, vol. 120, no. 20, pp. 9752-9762, 2004.

[25] F. F. Abraham, "A reexamination of homogeneous nucleation theory: thermodynamic aspects," Journal of the Atmospheric Sciences, vol. 25, pp. 47-53, 1998.

[26] R. C. Cammarata, "Generalized surface thermodynamics with application to nucleation," Philosophical Magazine, vol. 88, no. 6, pp. 927-948, 2008.

[27] F. G. Shi, "Direct measurement of free-energy barrier to nucleation of crystallites in amorphous silicon thin films," Journal of Applied Physics, vol. 76, no. 9, pp. 5149-5153, 1994.

[28] R. B. McClurg and R. C. Flagan, "Critical comparison of droplet models in homogeneous nucleation theory," Journal of Colloid and Interface Science, vol. 201, no. 2, pp. 194-199, 1998.

[29] A. Laaksonen, V. Talanquer, and D. W. Oxtoby, "Nucleation: measurements, theory, and atmospheric applications," Annual Review of Physical Chemistry, vol. 46, no. 1, pp. 489-524, 1995.

[30] R. Evans, "The nature of the liquid-vapour interface and other topics in the statistical mechanics of non-uniform, classical fluids," Advances in Physics, vol. 28, no. 2, pp. 143-200, 1979.

[31] S. Ryu and W. Cai, "Validity of classical nucleation theory for Ising models," Physical Review E-Statistical, Nonlinear, and Soft Matter Physics, vol. 81, no. 3, Article ID 030601, 2010.

[32] D. T. Wu, "Nucleation Theory," Solid State Physics, vol. 50, pp. 37-187, 1996.

[33] B. N. Hale, "Scaling of nucleation rates," Metallurgical Transactions A, vol. 23, no. 7, pp. 1863-1868, 1992.

[34] D. Stauffer, "Scaling theory of percolation clusters," Physics Reports, vol. 54, no. 1, pp. 1-74, 1979.

[35] K. R. Bauchspiess and D. Stauffer, "Use of percolation clusters in nucleation theory," Journal of Aerosol Science, vol. 9, no. 6, pp. 567-577, 1978.

[36] Y. S. Djikaev, R. Bowles, and H. Reiss, "Role of constraints in the thermodynamics of heterogeneous condensation on solid soluble particles: failure of the capillarity approximation," Physica A: Statistical Mechanics and Its Applications, vol. 298, no. 1-2, pp. 155-176, 2001.

[37] F. F. Abraham and G. M. Pound, "A reexamination of the free energy of droplet formation and dependence of surface tension on radius," Journal of Crystal Growth, vol. 6, no. 4, pp. 309-310, 1970.

[38] F. F. Abraham and G. M. Pound, "Free energy of formation of droplets from vapor and dependence of surface tension on radius," Journal of Crystal Growth, vol. 2, no. 3, pp. 165-168, 1968.

[39] W. R. Wilcox, "The relation between classical nucleation theory and the solubility of small particles," Journal of Crystal Growth, vol. 26, no. 1, pp. 153-154, 1974. 
[40] W. A. Cooper and C. A. Knight, "Heterogeneous nucleation by small liquid particles," Journal of Aerosol Science, vol. 6, no. 3-4, pp. 213-221, 1975.

[41] D. Schwarz, R. van Gastel, H. J. W. Zandvliet, and B. Poelsema, "Size fluctuations of near critical nuclei and gibbs free energy for nucleation of BDA on $\mathrm{Cu}(001)$," Physical Review Letters, vol. 109, no. 1, Article ID 016101, 2012.

[42] I. Kusaka, M. Talreja, and D. L. Tomasko, "Beyond classical theory: predicting the free energy barrier of bubble nucleation in polymer foaming," AIChE Journal, vol. 59, no. 8, pp. 30423053, 2013.

[43] H. Reiss and W. K. Kegel, "Replacement free energy and the 1/S factor in nucleation theory as a consequence of mixing entropy," Journal of Physical Chemistry, vol. 100, no. 24, pp. 10428-10432, 1996.

[44] D. Reguera and J. M. Rubi, "Nonequilibrium translationalrotational effects in nucleation," The Journal of Chemical Physics, vol. 115 , no. 15 , p. $7100,2001$.

[45] A. Reinhardt and J. P. K. Doye, "Free energy landscapes for homogeneous nucleation of ice for a monatomic water model," The Journal of Chemical Physics, vol. 136, no. 5, Article ID 054501, 2012.

[46] G. O. Berim and E. Ruckenstein, "Kinetic theory of heterogeneous nucleation; effect of nonuniform density in the nuclei," Journal of Colloid and Interface Science, vol. 355, no. 1, pp. 259264, 2011.

[47] R. McGraw and H. Reiss, "Nucleation near a critical temperature," Journal of Statistical Physics, vol. 20, no. 4, pp. 385-413, 1979.

[48] V. B. Kurasov, "Kinetic theory for condensation of multicomponent vapor under dynamic conditions," Physica A, vol. 207, no. 4, pp. 541-560, 1994. 

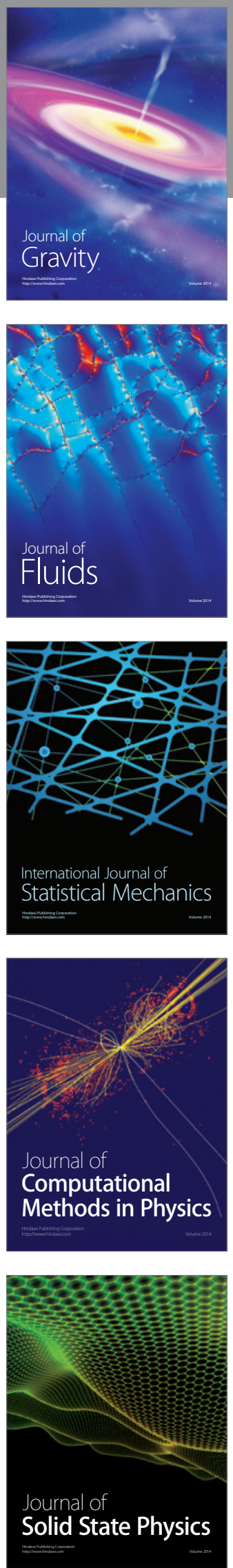

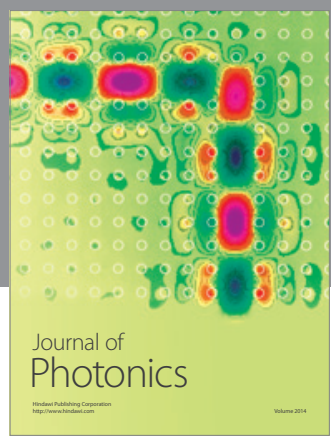

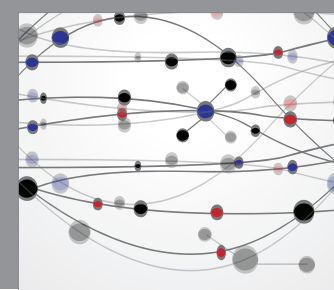

The Scientific World Journal

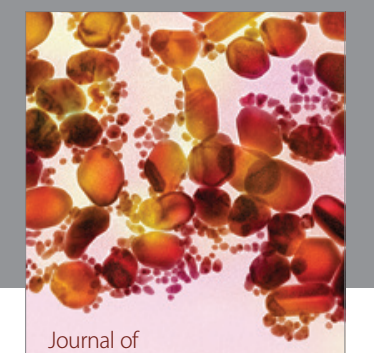

Soft Matter
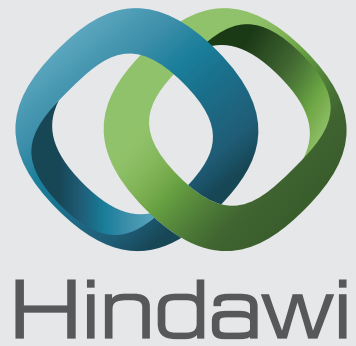

Submit your manuscripts at

http://www.hindawi.com
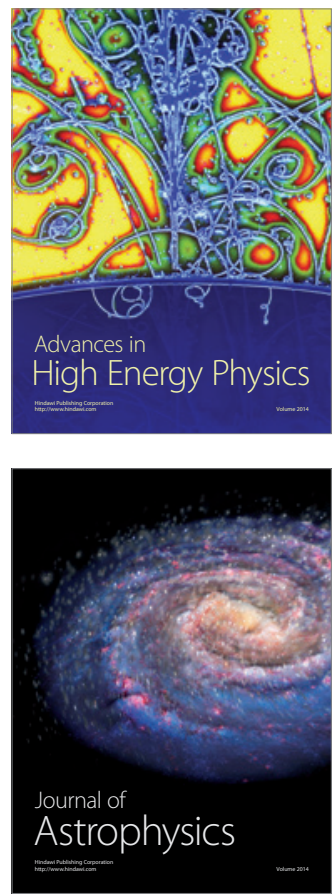
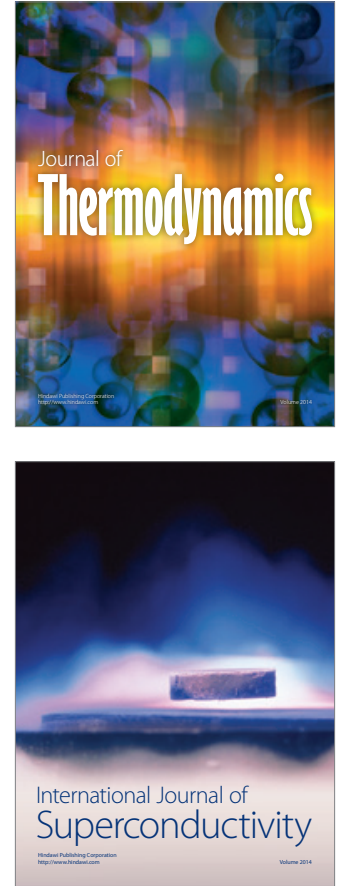
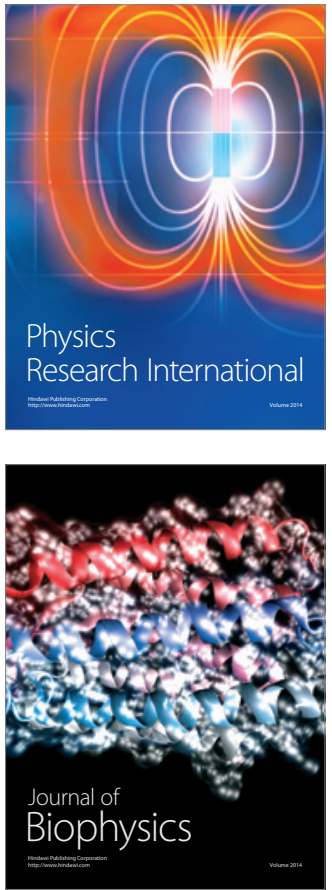
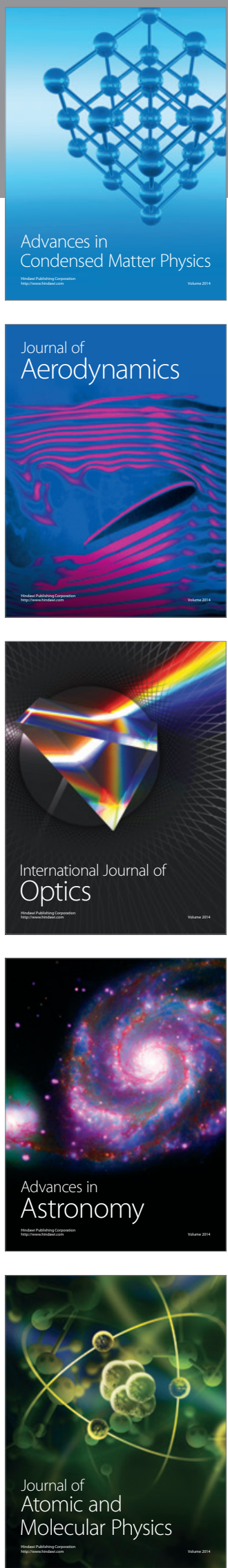